\title{
Research on Zigbee Indoor Position Algorithm Based on Particle Filter
}

\author{
Wu Wenhao 1 \\ 1.School of Information Science and Engineering, Central South University, Changsha, 410083 \\ China
}

Keywords: Zigbee Radio Frequency Communication Technology; Indoor Position; Received Signal Strength; Fingerprint Database; Particle Filter

\begin{abstract}
In an indoor environment, such outdoor positioning technology as GPS may have the phenomena of weak signal strength and poor availability due to construction blocking. As a result, the application of Zigbee wireless sensor network becomes a hotspot of the indoor position research at present. Specifically, the received signal strength of the nodes in Zigbee network can be used for providing location-based service, and then the fingerprint database position algorithm can be used for estimating user location on the basis of the experimental environment analysis. In order to improve position accuracy, the particle filter technology is combined with the fingerprint database position algorithm for improving the performance of the position system. The experiment shows that the optimized position algorithm can not only control the mean position error within $1.61 \mathrm{~m}$ to improve the position accuracy, but also has simple operation and great feasibility.
\end{abstract}

\section{Introduction}

Position accuracy is an important index for evaluating the performance of an indoor position system. Particle filter is a nonlinear filter method based on Bayesian estimation thought and can be used to solve nonlinear and non-Gaussian distribution problems. Since RSS is a nonlinear function [4] related to the measured target node location and the noise distribution in the complex indoor environment is the non-Gaussian distribution inconsistent with $N\left(\mu, \sigma^{2}\right)$, the radio frequency signal has irregular jump. In this article, the improved algorithm combined with fingerprint database and particle filter is adopted to reduce the influence of the above phenomenon on the position result.

\section{Indoor Position Algorithm Based on Particle Filter}

According to relevant research conclusions, RSSI based fingerprint database matching algorithm has been proven to become the main algorithm for present indoor position research[5]. The fingerprint database position algorithm process includes training stage and location stage, wherein the training stage aims at establishing RSS signal strength information database of the transmitting nodes at the beacon node; the location stage aims at comparing RSS of the transmitting nodes received by the target node and RSS of the beacon nodes in the fingerprint database established in the training stage in order to estimate the location of the target node. The algorithm execution process is as shown in Figure (1).

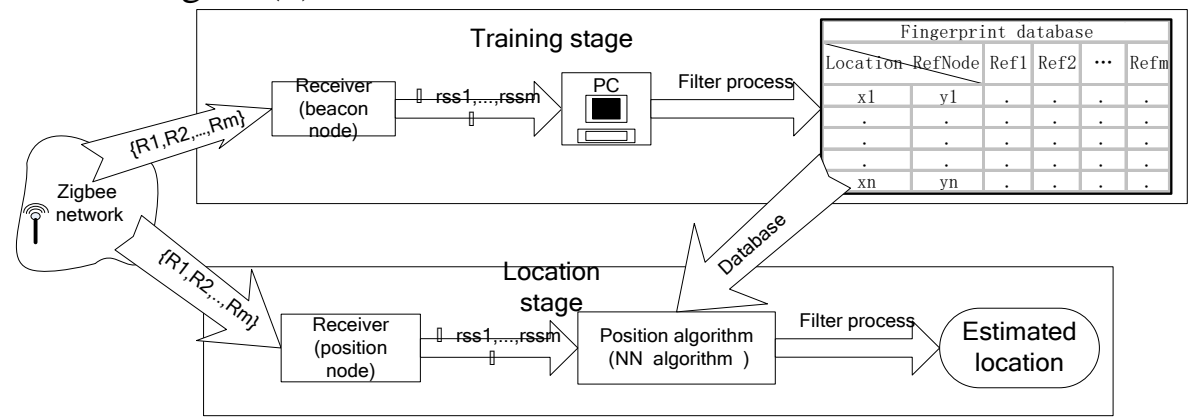

Fig.1. Fingerprint Database Algorithm Execution Process 
In the location stage, the target node receives the radio frequency signal strength from various transmitting nodes while moving in the position area, and then the above radio frequency signal strength is compared with RSS vector of various reference nodes in the fingerprint database established in the training stage in order to obtain the location of the target node through calculation. Meanwhile, nearest neighbor (NN) algorithm is adopted in this article to estimate the location of the target node.

As a relatively mature theoretical algorithm, NN algorithm makes use of RSS vector $R_{i} \in\left\{R_{1}, R_{2}, \cdots, R_{n}\right\}$, ( $\left.i \in[1, n]\right)$ of $\mathrm{n}$ reference nodes in the fingerprint database established in the training stage. In the location stage, the target node enters the position area at time $t$ and receives real-time RSS from $\mathrm{m}$ transmitting nodes, namely $r_{t}=\left\{\operatorname{rss}_{1}^{t}, \operatorname{rss}_{2}^{t}, \operatorname{rss}_{3}^{t} r s s_{4}^{t}\right\}$. Euclidean distance is selected to represent the neighbor degree of the target node and the beacon nodes in the fingerprint database in order to estimate the user location information at time t. The calculation formula is as shown in Formula (1):

$$
D_{n}=\sqrt{\sum_{i=1}^{m}\left(R S S_{i}-r S S_{i}^{t}\right)^{2}}
$$

$L=\min D_{n}\left(r_{t}, R_{n}\right)$ is set, namely: the location coordinates of the beacon node corresponding to the minimum Euclidean distance is the initial estimated location $S_{t, f p}$ of the target node at time $t$.

However, in actual test stage, some ineffective signal strength data affected by noise interference are also included in the position result calculation. In order to eliminate the influence of such ineffective information on position accuracy, it is necessary to implement the filter process for the initial position coordinates.

\section{Experiment Result}

Zigbee wireless communication network composed of CC2530 chips manufactured by TI Corporation is selected for the experiment to realize the communication among the nodes. A605 laboratory is selected as the experiment location, wherein the size of the experiment area is $7 \mathrm{mx} 7 \mathrm{~m}$, 64 beacon nodes (the distance between the reference nodes is $0.8 \mathrm{~m}$ ) and 4 transmitting nodes (they are arranged at the four corners of the experiment area, with the vertical distance of $2.3 \mathrm{~m}$ ) are respectively set in the above area. The node arrangement rule is as shown in Figure 2

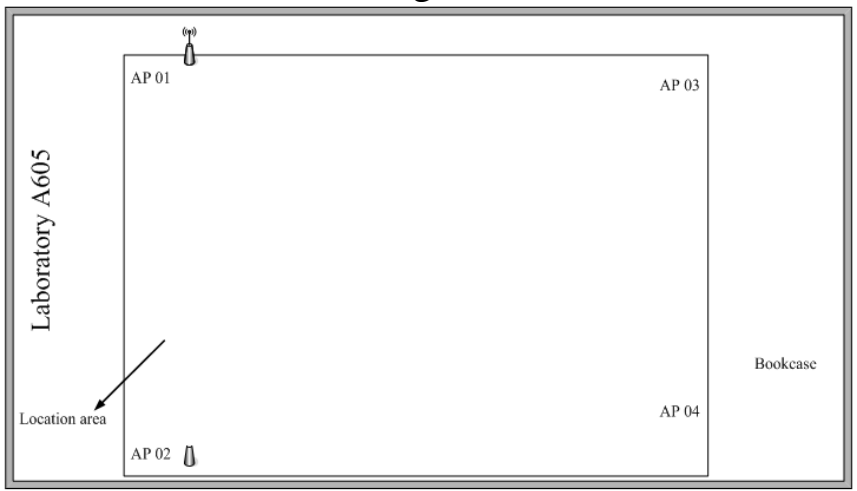

Fig.2. Experiment Area Setting and Node Arrangement

In order to maximally reduce the influence of such natural factors as weather on the experiment result, the sample strength information collected on March 31 is selected as the training data and the test data. In the online training stage, each reference node collects 160 groups of data, totally 10240 groups of data. In the offline location stage, 15 nodes are randomly selected as the static position test nodes (as the solid points shown in Figure (4)), and each node collects 25 groups of data, totally 375 groups of data.

In the static environment, the location coordinates of 15 static nodes are respectively obtained through calculation, and meanwhile the particle filter technology ( $\partial=0.8$ ) is adopted to smooth the initial position result.

The deviation and the mean position deviation of $1 \sim 15 \#$ static nodes are respectively as shown in 
Table 1.

Table 1 Position Error in Static Environment $(\partial=0.8)$

\begin{tabular}{cccc}
\hline & $\begin{array}{c}\text { Maximum Deviation } \\
(\text { Unit: m) }\end{array}$ & $\begin{array}{c}\text { Minimum Deviation } \\
(\text { Unit: m) }\end{array}$ & $\begin{array}{c}\text { Mean Deviation } \\
\text { (Unit: m) }\end{array}$ \\
\hline Fingerprint Database & 3.3252 & 0.4 & 1.9727 \\
$\begin{array}{c}\text { Fingerprint Database }+ \\
\text { Particle Filter }\end{array}$ & 3.0149 & 0.2918 & 1.6011 \\
\hline
\end{tabular}

According to Table (1), for the position result processed by particle filter, the minimum deviation can be reduced to 0.2918 , the mean position error can be controlled at about $1.6 \mathrm{~m}$ and the mean position accuracy is improved by $18.8 \%$. According to Figure (3), the improved algorithm has superior position result, and the position accuracy can be improved maximally by $84 \%$ (the 8th test node).

Through the cumulative distribution function (CDF) as shown in Figure 3 under two modes, we can know that compared with traditional fingerprint database algorithm, the improved algorithm combined with particle filter technology and fingerprint database has more reliable position result in the experiment area.

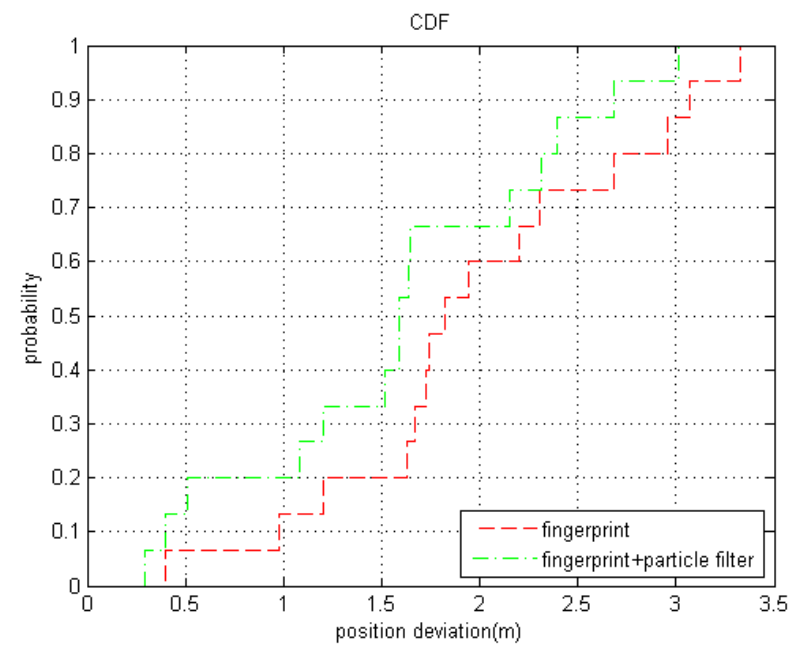

Fig.3. Position Error CDF Curve

Compared with the experiment result[6] in reference (6) (as shown in Table (2)), the improved algorithm can obtain similar position accuracy in the same-sized indoor scope, but the workload for reference node arrangement and fingerprint database establishment in the experiment is reduced by $11.1 \%$. Obviously, the position accuracy of the improved fingerprint database algorithm is improved more or less.

Table 2 Experiment Result Comparison

\begin{tabular}{|c|c|c|c|}
\hline & Experiment Aare (m) & Reference Node & Mean Deviation ( $\mathrm{m}$ ) \\
\hline $\begin{array}{c}\text { Result in References (NN } \\
\text { Algorithm, AP:4) }\end{array}$ & $6.7 * 6.8$ & $9 * 9$ & 1.6 \\
\hline $\begin{array}{l}\text { Result of the Algorithm in } \\
\text { this Article (AP:4) }\end{array}$ & $7 * 7$ & $8 * 8$ & 1.6011 \\
\hline
\end{tabular}

In dynamic experiment, the experimenter walks at a constant speed $\left(\mathrm{v}=6.67 \mathrm{~cm} / \mathrm{s}, \mathrm{a}=0 \mathrm{~cm} / \mathrm{s}^{2}\right)$ from the coordinate position $(40,560)$ to the coordinate position $(40,120)$. Due to the short test time in the dynamic test process, the quantity of the sample strength information obtained in the dynamic test environment is less than that obtained in static test environment. Therefore, in consideration of the actual test environment, the effective particle coefficient $\partial$ in the dynamic environment is set as 0.9. The experiment process is as shown in Figure 4. 


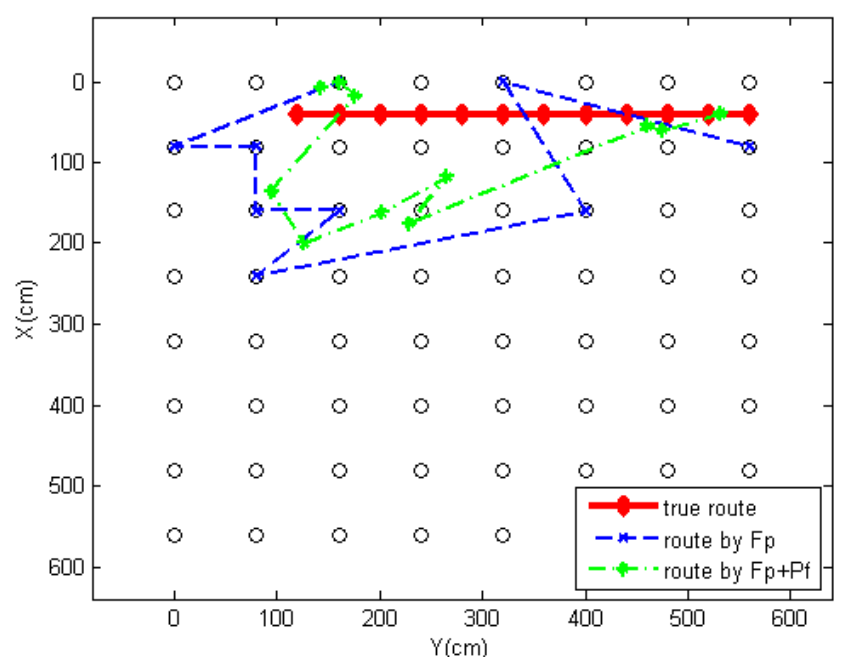

Fig.4. Result of Dynamic Experiment Based on Particle Filter $(\partial=0.9$ )

In Figure (7), the position error is the deviation between the position result obtained according to the indoor position algorithm and the true route. According to the above figure, compared with traditional fingerprint database algorithm (Fp), the improved algorithm combined with the particle filter ( $\mathrm{Pf}, \partial=0.9$ ) can obtain the position route more similar to the true route, thus able to achieve the expected effect of the experiment.

\section{Conclusion}

Under normal conditions, the indoor position algorithm based on RSS fingerprint database can provide relatively accurate position result. However, due to the influence of the external environment, RSS signal strength has jump phenomenon in the experiment process. The position algorithm combined with particle filter technology and fingerprint database can significantly reduce the influence of the radio frequency signal jump on the position result, and compared with traditional fingerprint database algorithm, this algorithm can effectively improve the position accuracy of the target node. Additionally, compared with other position modes, the fingerprint database position algorithm has relatively large workload. In consideration of the influence of the beacon node quantity on the position accuracy, the adoption of suitable interpolation algorithm for establishing fine grit database [4] is an important problem to be solved in future, and is also the common problem of the fingerprint database algorithm. 


\section{References}

[1] Li X, Lv Z, Hu J, et al. Traffic management and forecasting system based on 3d gis[J]. Cluster, Cloud and Grid Computing (CCGrid), 2015 15th IEEE/ACM International Symposium on, 2015: 991-998.

[2] Zhang S, Jing H. Fast log-Gabor-based nonlocal means image denoising methods[C]. Image Processing (ICIP), 2014 IEEE International Conference on. IEEE, 2014: 2724-2728.

[3] Jiang $\mathrm{D}, \mathrm{Xu} \mathrm{Z}$, Chen Z, et al. Joint time-frequency sparse estimation of large-scale network traffic[J]. Computer Networks, 2011, 55(15): 3533-3547.

[4] Wang K, Zhou X, Li T, et al. Optimizing load balancing and data-locality with data-aware scheduling[C]. Big Data (Big Data), 2014 IEEE International Conference on. IEEE, 2014: 119-128. 